\title{
Recordando a Sir William Osler a 100 años de su fallecimiento: ¿qué podemos aprender de su legado?
}

\author{
Remembering Sir William Osler 100 years after his death: what can we learn from
}

his legacy?

\author{
Robert E. Becker \\ Aristea Translational Medicine Corp, Carrabassett Valley, ME, USA.
}

An Fac med. 2015;76(1):71-6 / doi:10.15381/anales.v76i1.11078

"Yo fui nieto académico de Sir William Osler"

Andrews, 2002

William Osler es un médico por antonomasia en la historia de la medicina. Como padre de la medicina hipocrática moderna trasladó los cimientos de la educación médica del siglo XIX de los salones de clase a la cabecera de los pacientes (figura), introdujo el uso rutinario del laboratorio y la autopsia como parte de la educación y la práctica médica, y trajo consigo la escolaridad, humildad y la humanidad en la relación médico-paciente. Fue muy querido por quienes lo conocían y respetado por su generación y por las que la siguieron. Brindó atención al paciente preocupándose antes que todo por la persona misma. Osler aún recibe elogios -con justicia merecidos- por avanzar el sitial de la ciencia, de las humanidades y del profesionalismo en la medicina (véase la sección referencias). Osler es menos reconocido por el control de errores, la biología variacional, el método científico y el humanismo-asuntos problemáticos para la medicina moderna.

Para conservar sus raíces hipocráticas, la medicina moderna debe ser profesional en atender científica y humanamente a los pacientes y respaldar la investigación y el cuidado del paciente con prácticas asistenciales sólidas. A fines del siglo XX empezaron, uno después de otro, algunos problemas desestabilizadores que complicaron la práctica de la medicina. El número inadecuado de médicos generales y la amenaza de perder su profesionalismo devinieron reales para la medicina de los EE UU.

Hoy, la medicina está sujeta internacionalmente a diversos retos: regulaciones gubernamentales disfuncionales, costos descontrolados, seria morbimortalidad proveniente de los errores médicos, escasez de médicos en la atención primaria y -quizás el mayor presagio de las continuas dificultades en los EE UU- el fracaso de organizar el cuidado de la salud como un sistema de atención estable en lo económico y funcional.

La medicina tiene que respaldar el profesionalismo, tolerar y ser capaz de atemperar a aquellos que quieren hacerla o tratarla como un negocio, practicarla sin convertirla en un negocio, y justificar empíricamente la calidad de atención y su práctica en salud. La legislación, las regulaciones y los intereses especiales que socavan el profesionalismo no son de interés público. Para preservar el profesionalismo, la medicina requiere que el público comprenda que el respaldo del profesionalismo genera mejor salud y atención, así como reduce los costos. El futuro de la medicina es una avenida de doble sentido. Conforme se aproxima el centenario de su muerte, Osler nos aporta bases sólidas para preservar el profesionalismo médico. Nos enseña a hacer uso efectivo de la ciencia y el humanismo en la atención del paciente y la investigación, a merecer el respaldo de la gente, y manejar la atención en salud y las relaciones con el gobierno sin perder dicho profesionalismo.

William Osler (1849-1919) nació en la región rural de Ontario, Canadá, hijo de un clérigo anglicano. Formado en su niñez por tener acceso al microscopio, a estudios de polizoos y más adelante por su exposición a Sir Thomas Browne, Osler decidió estudiar medicina. Más adelante, él consideró que reducir la savia del arce a jarabe cuando era niño fue la preparación en su carrera para nuevas observaciones científicas en medicina. Después de publicar siendo un joven naturalista, Osler ingresó a la Escuela de Medicina de Toronto. Fascinado por la anatomía, los parásitos, las humanidades -aunque sin impresionarse con las clases brindadas en la facultad-, hizo su transferencia a McGill, para adquirir las experiencias a la cabecera del paciente que habían sido traídas de Edimburgo, Reino Unido. Después de su graduación, estudió en Europa por dos años antes de asociarse al staff de la facultad de medicina de McGill. Reconocido por sus conocimientos, en 1883 llegó a ser profesor en McGill y uno de los dos únicos fellows canadienses del Colegio Real de Médicos Británicos. En esta década de su graduación, Osler puso su sello en la 
educación médica de McGill que perduró durante el siglo XX. De manera similar, como profesor en Filadelfia y más adelante en Hopkins, revolucionó la educación médica de los EE UU al enseñar a los estudiantes en la cabecera del paciente.

En 1884, luego de lanzar una moneda al aire, Osler dejó Montreal por un centro más grande en la Universidad de Pensilvania. Después, en 1888, Osler aceptó con mucho entusiasmo la oportunidad de ser profesor en el planeado Hospital Johns Hopkins y Escuela de Medicina. En 1905, animado por su esposa de escaparse de su muy demandada práctica médica en Baltimore, aceptó el Profesorado Regio de Medicina en la Universidad de Oxford. Se quedó en Oxford hasta su fallecimiento en 1919. Osler, que nunca hizo evidente su eminencia a los demás, vivió con simplicidad, atendió a ricos y pobres, y no asistió a la ceremonia de su nominación como baronet (Sir) en 1911.

$\mathrm{Al}$ aprender, enseñar y practicar, Osler relacionó la clínica con el laboratorio, la sala de autopsia y la biblioteca. Los valores de Osler-aprender a la cabecera del paciente, la observación, inducción cautelosa, confirmación de las impresiones obtenidas con los pacientes en la biblioteca y el laboratorio, la ampliación del conocimiento cuando se correlaciona los hallazgos de la autopsia con las observaciones clínicas, y atender con igual cariño a cada persona, sin distinción de rango-aún definen para muchos el cómo debe ser practicada la medicina.

Osler admiró a Sydenham, el Hipócrates inglés, como "un hombre con muchas dudas." Persuadido por lo que podía y no podía aprender de sus pacientes, libros y laboratorios, Osler templó su entusiasmo por la ciencia con un escepticismo controlado. Esta visión condicionada enmarcó su práctica de la medicina. Transmitió su ejercer con preceptos prácticos y atemporales. Décadas más adelante, en McGill, aquellos que lo conocieron, como WW Francis y otros docentes que lo emularon, usaron sus preceptos para organizar el aprendizaje como estudiantes de medicina y después de su graduación. "Dejen que el paciente, con su historia y el examen físico, les brinde el diagnóstico." "Si no tienen un diagnóstico, repitan la anamnesis y el examen." "Usen los exámenes auxiliares para confirmar lo que han averiguado de su paciente." "En una epidemia, inmunicen y protejan a la población." "Todos los medicamentos son venenos. Los médicos los usan con beneficio de los pacientes." "Hagan el diagnóstico por exclusión." Osler nos enseña a cómo balancear en nuestro conocimiento lo que es conocido, lo no conocido y lo incierto.

Para Osler, la educación es "una preparación" "para reconocer que la verdad es difícil de conseguir, que los errores deben ser admitidos, lamentados y, sobre todo, que se debe aprender de ellos". La ciencia y el humanismo no pueden evitar incidentes originados por los errores; "Confíen tan poco como sea posible." Los riesgos de equivocarse no pueden ser separados de la adquisición y aplicación del conocimiento. "Empiecen con la convicción de que la verdad absoluta es difícil de alcanzar en asuntos que se relacionan con nuestros prójimos, sanos o enfermos, que las patinadas en la observación son inevitables aún con las facultades mejor entrenadas, que los errores de juicio ocurrirán en la práctica de un arte que consiste esencialmente en balancear las probabilidades." "El mejor doctor, como el general exitoso, es el que se equivoca menos". Evitar y corregir los errores representan la principal tarea en la medicina de Osler.

Osler observó la diversidad biológica en que una enfermedad puede ocurrir en un paciente determinado; de alguna manera es única en sus manifestaciones y curso clínico. Consideraba que en la investigación y en la práctica "La variabilidad es la ley de la vida, y así como no hay dos caras iguales, dos cuerpos tampoco se parecen y no hay dos individuos que reaccionen de la misma manera y que se comporten de forma parecida en las condiciones anormales que conoce- mos como enfermedad." Hoy día esta biología variacional reta a la medicina a ser genéticamente personalizada y, por la mayor evidencia de la importancia de la singularidad de las exposiciones al ambiente, es más amplia la comprensión científica de las enfermedades. El arte consistirá en considerar a cada persona como un individuo.

Osler reemplazó a William Pepper como Profesor de Medicina en la Universidad de Pensilvania. Como consecuencia de su curiosidad cuando era niño de algo que no se podía ver pero que estaba presente en el agua del estanque, Osler introdujo el primer microscopio en la práctica clínica en la escuela. Armado con sus estudios microscópicos en sangre, sus observaciones en la cabecera del paciente y los hallazgos de patología en la sala de autopsia, Osler fue pionero en enfatizar que la ciencia provee los métodos que conllevan al conocimiento en medicina.

La medicina científica moderna destaca el uso de la mejor evidencia disponible producto de la investigación. Osler y Pepper usaron los reportes de la literatura como la mejor evidencia. Esta aproximación a la evidencia asume implícitamente que son sólidos los métodos y condiciones para el desarrollo y la explicación de la mejor evidencia. Sin embargo, como lo reconoce la medicina basada en la evidencia moderna, "la búsqueda de la mejor respuesta actual" sufre potencialmente de errores en los criterios y en la investigación. El escepticismo de Osler se refiere a estas preocupaciones. Los métodos de Osler siguen la dirección de los investigadores de la ciencia básica moderna y de quienes desarrollan las nuevas tecnologías; ellos primero eliminan los errores para entonces permitir que procedan los experimentos y que las máquinas funcionen con seguridad. Osler aceptó la medicina hipocrática como científica, porque suscita los métodos que permiten asegurar la validez de la indagación que confirme y refine la mejor evidencia con los estudios clínicos y de laboratorio. 


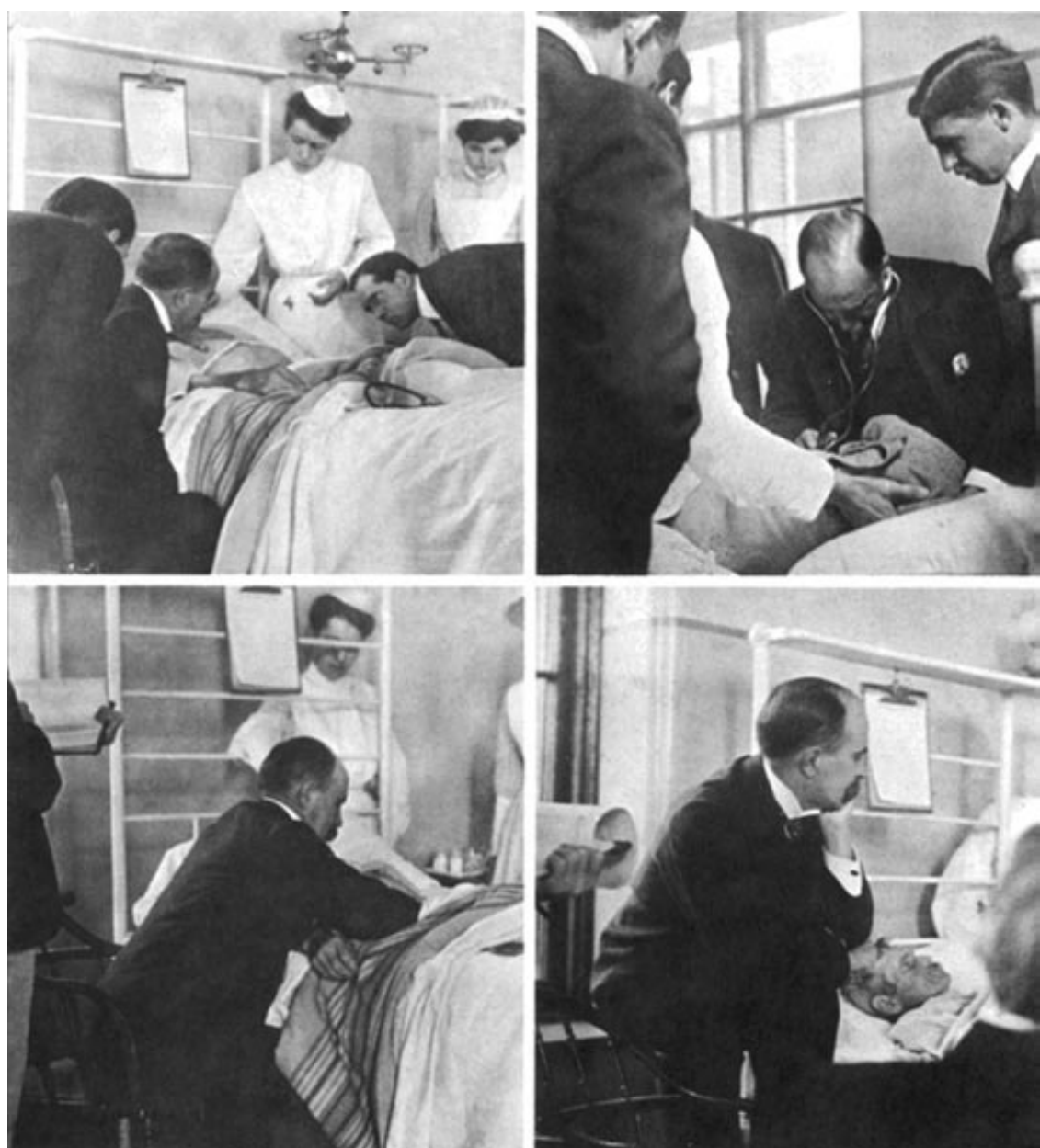

Figura 1. Sir William Osler a la cabecera del paciente.

Cushing da crédito a Pepper y su reconocido predecesor Stille de hacer a la Escuela de Medicina de Pensilvania famosa por las "brillantes y vívidas descripciones de una enfermedad". Pepper daba conferencias a los estudiantes de medicina regularmente, pero raramente aparecía en las salas de enfermos para enseñar a los estudiantes. En contraste, Osler estaba con los estudiantes en las salas de enfermos todos los días, con frecuencia enseñando en pacientes que nunca había visto antes y escogidos al azar. Osler hacía demostración de las enfermedades de los pacientes en las salas de conferencias y junto a la cabecera del enfermo. Con las observaciones del paciente, el microscopio y las autopsias, él correlacionaba las alteraciones patológicas con las historias clínicas y las anotaciones de los servicios. Desde el punto de vista de la educación médica de Pepper, uno aprendía sobre las enfermedades en los libros y de los expertos. Para Pepper, los pacientes ejemplificaban lo que se conocía de las enfermedades. Para Osler, los pacientes son la fuente en donde los médicos aprenden sobre las enfermedades.

Los métodos clínicos de Osler, al incorporar las respuestas a los errores médicos y las enseñanzas de la biología variacional de Darwin, personifican su idea de que los métodos representan la contribución organizadora de la ciencia a la medicina. Él reúne el conocimiento de la enfermedad, la fisiología, individualidad y otros adquiridos tanto del paciente como de los libros y el labo- ratorio, con las condiciones en que fueron obtenidos. En esta aproximación a la ciencia, Osler hace un paralelo con el físico contemporáneo suyo Pierre Duhem: "un experimento...no puede jamás condenar una hipótesis aislada, pero sí un supuesto teórico completo." Duhem consideraba que ninguna observación era válida si no se entendía cómo había sido hecha e interpretada. En el uso del método científico en medicina, Osler parece estar de acuerdo con Duhem, a quien aparentemente nunca leyó, de que la evidencia confirma como conocimiento o hecho todo conjunto de hipótesis, la hipótesis clínica o de investigación de interés primario y cualquier condición o método capaz de afectar el resultado y su interpretación. Los métodos de Osler abordan el mismo problema identificado por Duhem y con amplia influencia en la física moderna: "es perseguir una quimera el tratar de separar cada una de las hipótesis...de las otras suposiciones en la que esta ciencia reposa, de manera de someterla aisladamente a pruebas observacionales."

Los métodos de Osler privilegian las intervenciones contra la incertidumbre como centro de la actividad científica en medicina. Él interviene preventiva y retrospectivamente contra los errores, de manera similar a Duhem, a los físicos modernos y la industria. Duhem y Osler consideran que todo conocimiento y prácticas científicas pueden ser aceptadas condicionalmente, considerando siempre los métodos y las condiciones en que fueron adquiridas y la capacidad de ser puestas a prueba por la investigación y sus aplicaciones.

Osler enseñó medicina en los servicios -su epitafio- porque esa era la ruta apropiada para aprender medicina y porque su naturaleza era preocuparse por los demás. Él escribió "Nada te respaldará más...que el poder reconocer la verdadera poesía de la vida-la poesía del lugar común, del hombre ordinario, de la mujer simple cansada, con sus amores y alegrías, sus tristezas y sus penas." Fue conocido por tomar los pasos necesarios para satisfacer las ne- 
cesidades de cada paciente de manera individual. En los hechos, Osler definió el alcance del humanismo hipocrático.

El humanismo hipocrático admiteque el tema de la medicina es la persona que busca alivio de las tenazas de la enfermedad y del sufrimiento personal. Este humanismo reconoce el lazo entre el paciente y el doctor, reflejado en lo que ocurre cuando un niño pregunta y el doctor responde: "Doctor, doctor, imoriré?" "Sí, mi niño, y yo también moriré." El médico tiene una empatía natural con la situación de su paciente y busca aliviar, no aumentar, las preocupaciones del paciente cuando ve al doctor. Osler alivia a sus pacientes estableciendo una relación amigable y afable, como la tuvo con sus colegas y amigos. Como colega, Osler inspiró un amor de hermandad en la comunidad médica de Maryland. Osler fue un hombre de escritos y amante de los libros, con mucho conocimiento en humanidades y admirado por sus conocimientos, encanto, compasión y modestia.

Los médicos de Hopkins que lo conocían encontraban en Osler el significado de la cultura, erudición, carácter, humildad, humor y humanidad. Otros veían que Osler vivía sus enseñanzas: amor por sus semejantes, compasión por la gente enferma y oprimida, y una vida honesta y virtuosa. Osler unió el humanismo hipocrático -en el que uno llega a amar el bien y las posibilidades de bien para la humanidad- con el aprender. El aprendizaje del médico se dirige principalmente al conocimiento y los valores que se pueden obtener empíricamente en la atención de pacientes.

Los componentes fundamentales de Osler sobre la práctica hipocrática moderna-controles efectivos del error, biología variacional, métodos científicos, humanismo-hacen énfasis en problemas centrales que hoy amenazan la atención del paciente. Primero, durante la década pasada la medicina moderna no ha podido implementar las defensas sistemáticas, a profundidad y redundantes contra el error, como las que son necesarias para evitar los percances en la aviación y otros y recomendadas en medicina por el Instituto de Medicina de los EE UU en el 2000. Las muertes debidas a errores médicos continúan en todos los países desarrollados.

Segundo, el acceso clínico de la medicina a la individualidad del paciente ha disminuido. Un desbalance entre las especialidades tecnológicas y la atención primaria, manejo de las políticas de reembolso de la atención y el énfasis en una investigación insuficiente de los estudiantes de medicina en la atención del paciente, carga a los médicos con costos administrativos y reduce el tiempo dedicado en la atención integral del paciente. La medicina no puede respaldar que los estudiantes hagan especialidades, con aumento de los costos, si no enseña y persuade a la sociedad la necesidad de proveer la atención integral del paciente.

Tercero, la desinformación de las guías fundamentadas en la medicina basada en la evidencia arriesga la morbilidad y la mortalidad. Osler establece que la primera tarea de la medicina es ser científica en confirmar y reconfirmar la validez de la fuente utilizada para obtener o usar el conocimiento. Su tratado limpió la leña muerta para dejar el camino libre para el "advenimiento de la medicina científica". Solo entonces se condicionará apropiadamente los descubrimientos, traslaciones y cuidado del paciente a los riesgos del error. Con este contexto escéptico en el uso de la ciencia, Osler aprendió de los pacientes la utilidad variacional de los diagnósticos y tratamientos.

Cuarto, el empleo de pacientes simulados con diagnósticos hipotéticos como plataformas de lanzamiento para la educación en el cuidado del paciente falla en discriminar que el paciente simulado más entrenado transmite solo las manifestaciones típicas de la enfermedad. Las simulaciones no pueden desarrollar las habilidades aspiradas por Osler de aprender la biología variacional de las enfermedades, de los trata- mientos y de los pacientes, es decir, la biología individualizada de los pacientes.

El mensaje de Osler es que la manera como practicamos la medicina garantiza nuestro profesionalismo. Las apariencias de profesionalismo de la medicina, como lo identifica Cassell, provienen de los compromisos hipocráticos de los médicos. Osler señala una prioridad balanceada entre la responsabilidad científica y humanística. Usa las tecnologías y la empresa en servicio de la medicina y rechaza las intrusiones que comprometen a la medicina. Así como Osler, Duhem y los científicos de las ciencias básicas modernas comprenden que la ciencia no es una autopista al descubrimiento, sino un camino rocoso y con frecuencia serpenteante para llegar a disminuir la incertidumbre del conocimiento. Consistente con esta visión, Osler nos haría refinar, expandir $y$, en casos selectos, redireccionar las innovaciones médicas modernas.

La medicina necesita un programa nacional para eliminar los errores médicos. La medicina requiere políticas gubernamentales que apoyen el cuidado del paciente. El control solo de los costos reduce tanto los costos como la calidad de la atención medida por el tiempo que un médico dedica a un paciente. Los costos fijos y la competencia por la calidad pueden mejorar la calidad de atención al paciente. La medicina requiere de un sistema estable que permita ofrecer esta alta calidad de atención en salud, y no reparos con parches que quedaron anticuados cuando Bismarck introdujo el aseguramiento universal de la salud en Alemania, en 1883. Osler, conocido por no ocultar sus puntos de vista sobre el cuidado del paciente, haría que la medicina hablara fuerte su verdad a los gobiernos y al público.

La medicina necesita tanto la evidencia como los pacientes simulados para evolucionar y atacar más directamente los problemas planteados por la incertidumbre de los errores y la variación biológica. Un mayor énfasis en el aprendizaje observacional en 
la cabecera del paciente pondría a la medicina basada en la evidencia y las simulaciones en una perspectiva con mayor resonancia, restauraría el significado preeminente de las historias de los pacientes, de los exámenes físicos informativos y del cuidado de la persona, e impulsaría la tarea primaria de la medicina de asegurar la calidad de los recursos al servicio de la gente enferma.

Actuar para mejorar un problema presente implicaría intervenir contra cada uno de ellos. Por ejemplo, la decisión de un médico de brindar una atención observacional hipocrática de la enfermedad y de la persona en la cabecera del paciente no es posible hacerlo en los 10 a 20 minutos que tiene el profesional para una visita médica en los EE UU. Osler no esperaba que la medicina resolviera los problemas abrumadores impuestos sobre ella por la sociedad ni que se quedara en silencio. Osler pide a los médicos modelar y defender las prácticas derivadas de la tradición hipocrática. Corrigió lo que estaba a su alcance personal y no sucumbió a las presiones externas de desprofesionalización. Expresó sus objeciones a viva voz, pero no asumió que él pudiera siempre persuadir a los demás. Practicó lo que decidió practicar en las buenas y malas épocas, quién sabe ese sea su ejemplo esperanzador para todos los tiempos.

La medicina no puede siempre persuadir a los demás. Para alguna gente involucrada en la medicina, "la gente consiste en números...la ganancia de dinero es lo que reina". Osler no ofendió a aquellos con quienes no estaba de acuerdo, pero aceptaba que otros pudieran sentirse ofendidos por lo que decía. Él expresó la verdad impopular y era apreciado por hacerlo. Como Osler y otros han hecho énfasis, la medicina es un llamado personal humilde pero noble para quienes eligen hacerla así.

Los problemas de hoy son desalentadores. En la medicina uno no sufrirá si aprecia el bienestar, la vida misma y la muerte en paz al final de la vida que los médicos proporcionamos a cada pa- ciente. En un tiempo en que existían menos formas de alivio que ofrecer a los pacientes, Osler se sentía mejor cuando estaba en la cabecera del paciente de una pobre alma anciana con una enfermedad sin esperanza. Nosotros en medicina debemos transmitir al público que de esta manera la medicina hipocrática se puede mantener y proveer una atención de alta calidad. El público proporciona las condiciones que permiten tal atención.

Durante el pasado medio siglo, los avances científicos de la medicina han florecido, mientras las innovaciones y prácticas consistentes con la atención hipocrática integral del paciente han languidecido. Osler nos habría animado a practicar la medicina con la ecuanimidad y homeostasis biológica expresada en la tradición hipocrática, en lugar de sufrir las inestabilidades con las que vivimos hoy. En esta edad de tecnología en aumento, él nos guía a cómo usar estos nuevos recursos en la atención del paciente. Propongo que cada uno de nosotros resuelva provechosamente, con lo mejor de nuestras habilidades, vivir a la altura del legado de Osler, el Hipócrates canadiense, y saludar el centenario del fallecimiento de Osler con un gesto personal para afrontar el compromiso actual hacia la excelencia hipocrática.

\section{DECLARACIÓN DE INTERESES}

La preparación del artículo fue realizada por REB solamente. REB no ha recibido ayuda financiera para este trabajo y no tiene conflicto de intereses con relación a esta obra.

\section{TRADUCCIÓN}

Dr. José Pacheco.

\section{REFERENCIAS BIBLIOGRÁFICAS}

1. Andrews BF. Sir William Osler's emphasis on physical diagnosis and listening to symptoms. South Med J. 2002;95:1173-7.

2. Golden RL. William Osler at 150. An overview of a life. JAMA. 1999;282:2252-8.
3. Cushing, $\mathrm{H}$. The Life of Sir William Osler. New York: Oxford University Press; 1940.

4. Kaynes G. The Oslerian Tradition. BMJ. 1968;4:599604.

5. Lundberg G. Countdown to the Millennium-Balancing the Professionalism and Business of Medicine: Medicine's Rocking Horse. JAMA. 1990;263:86-7.

6. Relman AS. Shattuck Lecture--the health care industry: where is it taking us? N Engl J Med. 1991;325:854-9.

7. Goldberg RM. What's happened to the healing process? The Wall Street Journal. June 18, 1997: A22.

8. Kohn LT, Corrigan JM, Donaldson M, S Institute of Medicine. To Err Is Human: Building a Safer Health System. Washington: National Academy Press; 2000.

9. General Medical Council. The state of medical education and practice in the UK report: 2013. http:// www.gmc-uk.org/publications/23435.asp Accessed Aug 1, 2014.

10. Hall S. Medical error death risk 1 in 300. The Guardian, 2006; http://www.theguardian.com/society/2006/ nov/07/health.lifeandhealth Accessed Aug 1, 2014.

11. The College of Emergency Medicine. A call for action to get the NHS finance system to stop penalising Emergency Departments. 28 July 2014; http://www. collemergencymed.ac.uk/Public/Latest\%20News/ CEM\%20Statements Accessed Aug 1, 2014.

12. Bell L. Why the Centers for Disease Control should not receive gun research funding. 2013; http:// www.forbes.com/sites/larrybell/2013/02/12/why-thecenters-for-diseasecontrol-should-not-receive-gunresearch-funding/ Accessed Aug 1, 2014.

13. Berwick DM, Nolan TW, Whittington J. The Triple Aim: Care, Health, And Cost. Health Aff. 2008;27:759-69.

14. Bean RB, Bean WB. Sir William Osler: Aphorisms from His Bedside Teachings and Writings. Springfield (IL): Charles C Thomas; 1968.

15. Osler W. The Student Life in The Medical News ( 30 September 1905). In: Osler, W. ed. Aequanimitas with Other Addresses to Medical Students, Nurses and Practitioners of Medicine. Philadelphia, Pa.; P. Blakiston's Son \& Co.; 1925:413- 44.

16. Osler W. Teacher and student. In: Osler, W. ed. Aequanimitas with Other Addresses to Medical Students, Nurses and Practitioners of Medicine. Philadelphia, Pa.; P. Blakiston's Son \& Co.; 1925:21-44.

17. Osler, W. The pathological institute of a general hospital. Glasgow Medical Journal. 1911;76:321-33.

18. Osler W. On the educational value of the medical society. In: Osler, W. ed. Aequanimitas with Other Addresses to Medical Students, Nurses and Practitioners of Medicine. Philadelphia, Pa.; P. Blakiston's Son \& Co.; 1925:343-62.

19. Sackett DL, Straus SE, Richardson WS, Rosenberg W, Jaynes RB. Evidence-Based Medicine. How to Practice and Teach EBM. New York: Churchill Livingston; 1997.

20. Prasad V, Vandross A, Toomey C, Cheung M, Rho J, Quinn S, Chacko SJ, Borkar D, Gall V, Sevaraj S, Ho N, Cifu A. Decade of reversal: an analysis of 146 contradicted medical practices. Mayo Clin Proc. 2013;88:790-8

21. Doshi P, Dickersin K, Healy D, Vedula SS, Jefferson T. Restoring invisible and abandoned trials: a call for people to publish the findings. BMJ. 2013;346:f2865

22. Reason J. Human Error. Cambridge (UK): Cambridge University Press; 2001.

23. Maynard WB. Nothing succeeds like failure. Princeton Alumni Weekly, April 7, 2009:18-23.

24. Duhem P. The Aim and Structure of Physical Theory. Princeton, NJ: Princeton University Press; 1954. 
25. Dyson FW, Eddington AS, Davidson C. A determination of the deflection of light by the sun's gravitational field, from observations made at the total eclipse of May 29, 1919. Philosophical Transactions of the Royal Society of London, Series A. 1920;220:291-333. http://astro.berkeley.edu/ kalas/labs/documents/ dyson1 920.pdf Accessed May 10, 2014.

26. Osler W. An Alabama Student. In: Osler, W. An Alabama Student and Other Biographical Essays. London: Oxford University Press; 1908:1-18.

27. Society for Academic Primary Care. New Century, New Challenges. A Report from the Heads of Departments of General Practice and Primary Care in the Medical Schools of the United Kingdom. September 2002 http://www.sapc.ac.uk/documents/ Mackenzie2.pdf

28. Cooper Z, Gibbons S, Jones S, McGuire A. Does hospital competition save lives: evidence from the English NHS Patient Choice Reform. The Economic Journal. 2011;121:F228-F260.

29. Field RJ. A time to heal. The Pharos Spring 1996;11-3.

30. Kelly M. Sir William Osler: Aphorisms from His Bedside Teachings and Writings. Arch Intern Med. 1963;111:264-5.
Articulo reproducido de The Lancet 2014; 384 . 2260-63. DOI: http://dx.doi.org/10.1016/S01406736(14)61887-0

Con autorización del autor y de Elsevier-Copyright Clearance Center's Rights Link service, License Number: 3540770130270

Correspondencia: Dr. Robert E Becker,

7123 Pinebrook Road, Park City,

UT 84098, USA

E-mail: rebecker2008@comcast.net 